\title{
A Novel Tool to Measure Extracellular Glutamate in the Zebrafish Nervous System In Vivo
}

\author{
Ryan B. MacDonald,, ${ }^{1,2}$ Nachiket D. Kashikar, \\ Leon Lagnado, and William A. Harris ${ }^{2}$
}

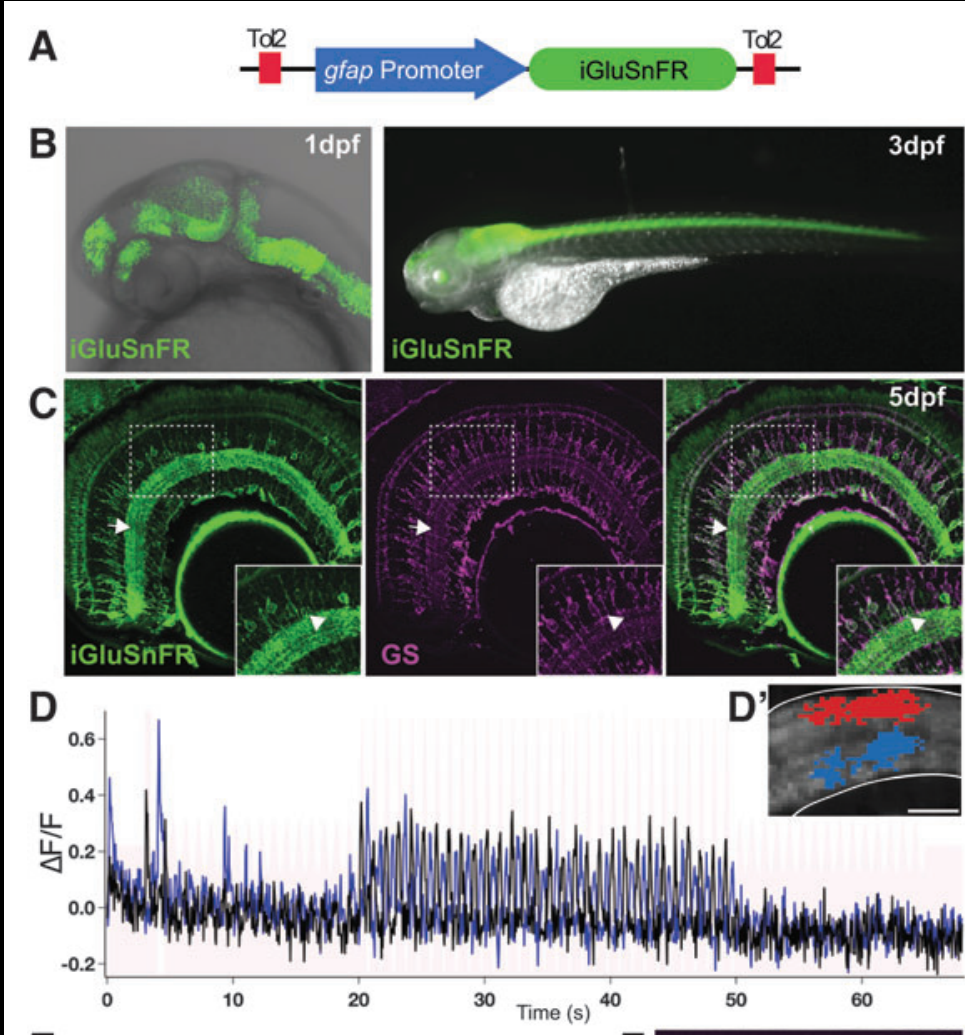

E

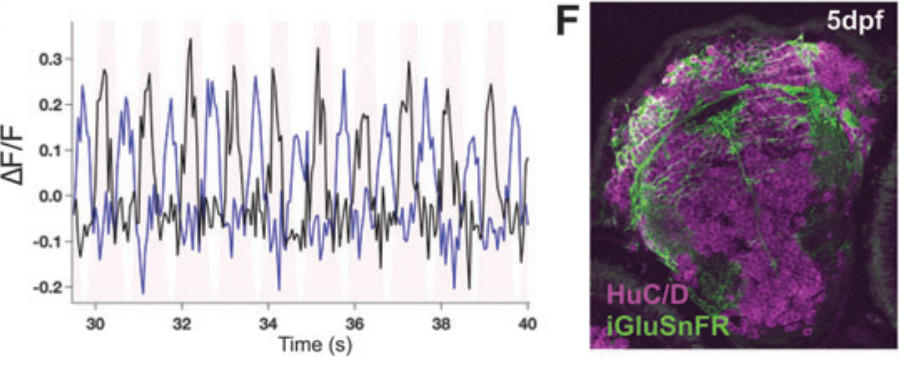

${ }^{1}$ Department of Infection, Immunity and Cardiovascular Disease, Medical School and the Bateson Centre, University of Sheffield, Sheffield, United Kingdom.

${ }^{2}$ Department of Physiology, Development and Neuroscience, University of Cambridge, Cambridge, United Kingdom.

${ }^{3}$ Sussex Neuroscience, School of Life Sciences, University of Sussex, Brighton, United Kingdom.

(C) Ryan B. MacDonald, et al., 2016; Published by Mary Ann Liebert, Inc. This Open Access article is distributed under the terms of the Creative Commons License (http://creative commons.org/licenses/by/4.0), which permits unrestricted use, distribution, and reproduction in any medium, provided the original work is properly credited. 


\begin{abstract}
Glutamate is the major excitatory neurotransmitter in the brain. Its release and eventual recycling are key to rapid sustained neural activity. We have paired the gfap promoter region with the glutamate reporter molecule, iGluSnFR, to drive expression in glial cells throughout the nervous system. $T g$ (gfap:iGluSnFR) is expressed on the glial membrane of Müller glia cells in the retina, which rapidly respond to stimulation and the release of extracellular glutamate. As glial cells are associated with most, if not all, synapses, $T g$ (gfap:iGluSnFR) is a novel and exciting tool to measure neuronal activity and extracellular glutamate dynamics in many regions of the nervous system.
\end{abstract}

Keywords: glia, retina, glutamate, nervous system

G LUTAMATE IS THE MAJOR excitatory neurotransmitter in the brain. Its release and eventual recycling are key to rapid sustained neural activity. ${ }^{1}$ Glial cells play a key role in the uptake and recycling of glutamate from the synaptic cleft. iGluSnFR has been used to study synaptic activity by measuring glutamate dynamics in the zebrafish nervous system. ${ }^{2,3}$ Previous work has also used iGluSnFR in glial cells; however, this was done transiently in the mouse using viral vectors. ${ }^{2,4}$ As such, we designed a transgene to stably express iGluSnFR in the glial cells of the zebrafish nervous system. We report a novel transgenic zebrafish, $\operatorname{Tg}($ gfap:iGluSnFR), that displays the glutamate-sensitive fluorescent reporter iGluSnFR specifically on the membrane of glial cells (Figure 1A-C). This molecule is expressed on the glial membrane in many brain regions and rapidly responds to stimulation and the release of extracellular glutamate (Figure 1D-F, Supplementary Data; Supplementary Data are available online at www.liebertpub.com/zeb). Thus, pairing the sensitivity of iGluSnFR and optical transparency of the zebrafish provides a powerful tool for understanding glutamate dynamics in neural tissues in vivo.

\title{
Acknowledgments
}

We thank Shin-Ichi Higashijima for the gfap:dTomato Tol2 plasmid and Loren Looger for the iGluSnFR construct. R.B.M. is funded by a JG Graves Medical Research Fellowship at the University of Sheffield. N.D.K.was funded by a Marie Curie (IEF) Fellowship (331610). L.L. is funded by Wellcome Trust (Grant 102905) and BBSRC (BB/L021528/1). W.A.H. is funded by the Wellcome Trust Investigator Award.

\section{Disclosure Statement}

No competing financial interests exist.

FIG. 1. (A) To express iGluSnFR specifically in glial cells, we used the gfap promoter region to generate the $T g(g f a p: i G l u S n F R)$ transgenic line. (B) Whole mount fluorescent images of the $T g(g f a p$ :iGluSnFR) embryos showing expression begins at 1 day postfertilization (dpf) in the embryonic and in the maturing nervous system at $3 \mathrm{dpf}$. (C) Sections showing iGluSnFR expression in the retinal Müller glia cells, labeled with the glutamine synthetase (GS) antibody, up to at least 5 dpf. Inset shows that all GS-positive cells are also iGluSnFR positive in the retina. The iGluSnFR signal is enriched in the inner plexiform layer (arrow) presumably because of the presence of low-level extracellular glutamate in the synaptic layer of the retina. There are occasional GS-negative cells, presumably neurons, expressing iGluSnFR in the inner nuclear layer of the retina (arrowhead). These cells may be labeled because of early activity of the gfap promoter in progenitors during the genesis of neurons, which has been noted previously. ${ }^{5}$ (D) iGluSnFR in the retina responds to light stimulation as characterized by activity-dependent changes in fluorescence over time in the ON (black plot) and OFF (blue plot) regions of the inner plexiform layer (arrow). (D') An example of the region of interest used to measure ON (blue) and OFF (red) responses in the inner plexiform layer of the retina. Scale bar denotes $10 \mu \mathrm{m}$. (E) Zoom of D between 30 and 40 s clearly shows the light-dependent responses in the retina. (F) iGluSnFR is also expressed in the glial cells and neuropil of the brain, but not the neurons (HuC/D positive) in sections at $5 \mathrm{dpf}$. 


\section{References}

1. Platt SR. The role of glutamate in central nervous system health and disease-a review. Vet J 2007;173:278-286.

2. Marvin JS, Borghuis BG, Tian L, Cichon J, Harnett MT, Akerboom J, et al. An optimized fluorescent probe for visualizing glutamate neurotransmission. Nat Methods 2013;10:162-170.

3. Zhang RW, Li XQ, Kawakami K, Du JL. Stereotyped initiation of retinal waves by bipolar cells via presynaptic NMDA autoreceptors. Nat Commun 2016;7:12650.

4. Borghuis BG, Looger LL, Tomita S, Demb JB. Kainate receptors mediate signaling in both transient and sustained OFF bipolar cell pathways in mouse retina. J Neurosci 2014;34:6128-6139.

5. Bernardos RL, Raymond PA. GFAP transgenic zebrafish. Gene Expr Patterns 2006; 6:1007-1013.

Address Correspondence to:

Ryan B. MacDonald, PhD

Department of Infection, Immunity and Cardiovascular Disease

Medical School and the Bateson Centre

University of Sheffield

Sheffield S10 2TN

United Kingdom

E-mail: r.macdonald@sheffield.ac.uk 